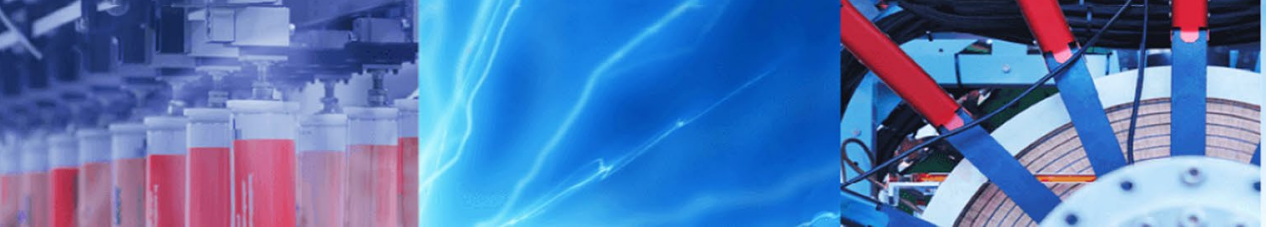

Research Article

\title{
Exploitation of the hard/soft segments ratio in thermoplastic polyurethane (TPU) for the tuning of electrical and mechanical properties of expanded graphite (EG) based composites
}

\author{
Francesco Piana $^{1,2,3}$ (D) Jürgen Pionteck ${ }^{1}$ (D)
}

C Springer Nature Switzerland AG 2019

\begin{abstract}
Composite materials based on three kinds of commercial thermoplastic polyurethanes (TPUs) and electrically conductive expanded graphite (EG) were prepared by melt-mixing. The TPUs differed mainly in hard/soft segment ratio, resulting in different polarity and crystallinity. These parameters were exploited to prepare composite materials with different percolating path structure, whose characteristics were thoroughly analysed. Test specimens were prepared by compression moulding and their electrical, thermal and mechanical properties, as dependent on filler content, were analysed. The electrical behaviour was correlated to the morphologies. The results showed how EG promoted and modified the crystallization, resulting in enhanced hardness but reduced toughness of the material. Moreover, EG segregation was observed in the TPU with the highest HS content which already promoted percolating paths at EG concentration below $2 \mathrm{wt} \%(1.1 \mathrm{vol} \%)$ and showed a resistivity between $10^{5}$ and $10^{4} \Omega \mathrm{cm}$, while in TPU with low crystallinity the percolation was shifted to above $4 \mathrm{wt} \% \mathrm{EG}$ and the resistivity reached values slightly over $10^{5} \Omega \mathrm{cm}$ at 8 and $10 \mathrm{wt} \% \mathrm{EG}$. These peculiarities enabled easy management of a broad range of industrial application such as antistatic coatings, mechanically reinforced insulators, and electrical conductive connectors using a few pristine materials.
\end{abstract}

Keywords Composites · Elastomers · Nanoparticles · Polyurethanes · Expanded graphite · Structure-property relations . Conductivity

\section{Introduction}

Composites materials loaded with conductive carbonbased filler were studied intensively in the last decades especially after the development of graphene [1] and carbon nanotubes [2] production. They immediately found applications in fields like flame retardants [3], thermal dissipative [4], antistatic [5] and reinforced materials [6] and then expanding to various electronic applications [7-9]. Highly dispersed graphite-like fillers in versatile polymer matrices like thermoplastic polyurethanes (TPUs) enabled interesting novel applications like phase-change [10,11] and shape memory $[12,13]$ materials. Insights into the interactions between the filler and the crystalline domains of the polymers are determinant for these and similar fields of application [14]. The evaluation of the influence of added expanded graphite (EG) on the morphological, electrical and mechanical properties of TPUs with different compositions in soft and hard segment contents (SS and $\mathrm{HS}$, respectively) provided important information that can promote the comprehension and applicability of these systems [15]. As an evidence of this importance, it was demonstrated that electric characteristics can be tuned by acting on the parameters that influence the crystallinity of the polymer matrix in polylactic acid/graphene nanoflakes [16].

$\triangle$ Francesco Piana, piana@imc.cas.cz | 'Leibniz-Institut für Polymerforschung Dresden e.V., Hohe Straße 6, 01069 Dresden, Germany. ${ }^{2}$ Organic Chemistry of Polymers, Technische Universität Dresden, 01062 Dresden, Germany. ${ }^{3}$ Present Address: Institute of Macromolecular Chemistry, Academy of Sciences of the Czech Republic v.v.i., Heyrovsky Sq. 2, 16206 Prague 6, Czech Republic.

SN Applied Sciences (2019) 1:878| https://doi.org/10.1007/s42452-019-0908-3 
Significant progress were made in reducing the cost of high-performance conductive carbon fillers like nanotubes, but expanded graphite is still a strong competitor in industrial use thanks to its availability [17], very low toxicity [18], and the simple, economical processes for producing [19] and converting it into the final product [20]. EG dispersed in polymer matrices above a certain concentration, the so-called percolation concentration (PC) increases the electrical conductivity $(\sigma)$ by many orders of magnitude [21]. When applying alternating current (ac) to the composite above this threshold composition, $\sigma^{\prime}$ and the permittivity $(\varepsilon)$ become nearly independent of frequency and temperature [22]. The PC is influenced in general by the geometry $[23,24]$ and dimension [25] of the filler and by the mixing method [26]. In composites prepared by melt-mixing, factors influencing the EG dispersion such as the viscosity of the medium [27], the presence of surfactants [28] and processing parameters [29] can shift the PC significantly.

In the present work we prepared three series of TPU/EG composites with EG concentration between 0 and $10 \mathrm{wt} \%$ using three commercial TPUs differing primarily in the HS/ SS ratio and SS-oligomers, polyether or polyester based. Elastollan ${ }^{\circ}$ S60A15 is a TPU with low HS concentration which contains a softener and is almost completely amorphous. Elastollan $1185 \mathrm{~A}$ is a TPU with intermediate HS/ SS ratio close to $1: 1$ and medium crystallinity which was already used in a previous work [27]. Elastollan ${ }^{\circ}$ C74D50 contains the highest HS amount and is highly crystalline. For the sake of simplification, the TPUs are named in the text as S60A15 or Ic-TPU, 1185A or mc-TPU, and C74D50 or hc-TPU, respectively. It must be noted that Elastollan $1185 \mathrm{~A}$ contains polyether diol as SS while the others are based on polyester diols. The higher difference in the surface tensions between HS and polyether SS compared to that of HS/polyester SS should result in stronger phase separation [30]. It was found that the different HS/SD ratios in these TPUs influence not only the composition itself but also that higher HS concentration provides a more pronounced phase separation with consequently higher crystallinity in HS domains [30]. In this work, the crystallinities of the three different TPUs and their composites with
EG have been investigated by DSC, verifying that the filler modifies the biphasic structure of TPUs. The dependency of the electrical conductivity on EG concentration was used to estimate $P C$ values and these results have been compared with the previous data determined for mc-TPU. To support the interpretation of the electrical measurements, the morphology at the TPU/EG interface has been observed by SEM.

The tendency of the amorphous phase composed prevalently of SS was found to envelop and insulate the graphite nanoplatelets (GNP). The different influence of EG on phase separation and the properties of the three biphasic TPU also became evident by TGA, where the presence of EG mainly affected the degradation of the SS phase, and by measuring the tensile behaviour and hardness and its dependence on composition.

\section{Experimental section}

\subsection{Materials}

Linear thermoplastic polyurethanes of the Elastollan ${ }^{\circ}$ series were obtained from Elastogran (BASF Group) (Table 1): low crystalline S60A15 (Ic-TPU) containing a softener, middle crystalline 1185A (mc-TPU), and high crystalline C74D50 (hc-TPU). Expanded graphite, SIGRAFLEX ${ }^{\circ}$ Expandat, has been provided from SGL Technologies $\mathrm{GmbH}, \mathrm{SGL}$ Group. Measured properties were: conductivity $\sigma=40 \mathrm{~S} / \mathrm{cm}$ (room temperature, $30 \mathrm{MPa}$, self-made 2-electrode powder conductivity tester, coupled with a DMM 2000 Electrometer, Keithley Instruments); apparent volume $\sim 400 \mathrm{~cm}^{3} / \mathrm{g}$; specific surface $39.4 \mathrm{~m}^{2} / \mathrm{g}\left(77.4 \mathrm{~K}, \mathrm{~N}_{2}\right.$ atmosphere, Autosorb-1, Quantachrome). The elemental composition of EG determined by quantitative X-ray photoelectron spectroscopy (XPS) using an AXIS ULTRA (Kratos Analytical, England) was [O]:[C] $=0.006$ and no $s p^{3}$ hybridization could be detected. Wide angle X-ray scattering (WAXS) performed with an XRD 3003 T/T (SeifertFPM Freiberg/Sa., Germany) provided a typical diffraction pattern corresponding well to that of pure graphite (but with broadened reflections due to the lower crystal size)

Table 1 Characteristic data for the used TPU, provided by the producer

\begin{tabular}{lllll}
\hline TPU & S60A15 (Ic-TPU) & $1185 A(\mathrm{mc}-\mathrm{TPU})$ & C74D50 (hc-TPU) & DIN \\
\hline Polyol & Ester & Ether & Ester & - \\
Additive & Softener & - & - & - \\
Hardness & 63 Shore A & 87 Shore A, 36 Shore D & 73 Shore D & ISO 7619-1 \\
Density $\left(\mathrm{g} / \mathrm{cm}^{3}\right)$ & 1.19 & 1.12 & 1.25 & EN ISO 1183-1-A \\
$\mathrm{E}_{\mathrm{t}}(\mathrm{MPa})$ & 35 & 45 & 45 & $53504-\mathrm{S} 2 /$ ISO 37 \\
Stress at 300\% strain $(\mathrm{MPa})$ & 6.5 & 10 & 35 & $53504-\mathrm{S} 2 /$ ISO 37 \\
\hline
\end{tabular}


and some small peaks of unknown substances, possibly impurities. Both methods prove the nearly complete graphitic structure of EG.

\subsection{Methods}

\subsubsection{Sample preparation}

The TPUs and EG were dried at $85^{\circ} \mathrm{C}$ under vacuum overnight before use. Composites were prepared via meltmixing using a twin screw DSM $5 \mathrm{ml}$ Micro-Compounder (Xplore, Sittard, The Netherlands). To obtain comparable shear forces, which strongly influence the filler dispersion [29], three different temperatures were applied: $160^{\circ} \mathrm{C}$ for LC-TPU, $200^{\circ} \mathrm{C}$ for mc-TPU and $240^{\circ} \mathrm{C}$ for HC-TPU. First the polymer was filled in the compounder and then the EG was added into the mixer. Four gram of material were mixed for $5 \mathrm{~min}$. under different processing conditions. The resulting product is a strand circa $1.5-2.5 \mathrm{~mm}$ in diameter, which was cooled in air on a metallic plate. For conductivity analysis, round plates $(25 \mathrm{~mm}$ in diameter, thickness $1.5 \mathrm{~mm}$ ) were prepared with an electrohydraulic bi-column press TYPE PW 40 EH (Paul-Otto Weber $\mathrm{GmbH}$, Remshalden, Germany). For that, strands produced via melt-mixing were cut to pellets and compression moulded at $100 \mathrm{kN}$ in air for $2 \mathrm{~min}$ at the same temperature used for melt-mixing. After reducing the temperature to circa $80^{\circ} \mathrm{C}$ within $5 \mathrm{~min}$. The sample was removed from the press and rapidly cooled to room temperature by putting it between pre-cooled metal plates. For mechanical tests, plates of the dimension $42 \times 40 \times 1 \mathrm{~mm}^{3}$ were compression moulded under the same conditions and dog bone shaped specimen with an effective gauge dimension of $20 \times 2 \times 1 \mathrm{~mm}^{3}$ were cut from it for tensile testing.

\subsubsection{Resistivity determination}

Volume resistance measurements were carried out on a 6175A Electrometer (Keithley Instruments, Solon, Ohio), coupled with an 8009 Resistivity Test Fixture with two plate electrodes located on both sides of the samples. The measurements were repeated on both sides of two specimens. According to ASTM D257-07 this method is suitable for resistance values in the range from $10^{7}$ to $10^{18} \Omega$.

\subsubsection{Morphological analysis}

Scanning electron microscopy (SEM) was used to analyse the microstructure of pure EG and TPU/EG composites by an SEM Supra 55VPO (Carl Zeiss NTS GmbH, Oberkochen, Germany). Strands and plates were cooled with liquid nitrogen, fractured, and then the fractured surface was sputtered with Pt to hinder electrostatic charging during SEM analysis.

\subsubsection{Thermal analysis}

Differential Scanning Calorimetry (DSC) and ThermoGravimetrical Analysis (TGA) were performed on a aliquot of 5-10 mg samples using a DSC Q 1000 and a TGA Q5000, respectively (both from TA Instruments, New Castle, Delaware). DSC was done in $\mathrm{N}_{2}$ atmosphere: 1 st heating from room temperature to $250^{\circ} \mathrm{C}$ at the scan rate of $10 \mathrm{~K} / \mathrm{min}$, cooling from 250 to $-20^{\circ} \mathrm{C}$ at $10 \mathrm{~K} / \mathrm{min}$ followed by fast cooling at $-80^{\circ} \mathrm{C}$ and 2 nd heating from -80 to $250^{\circ} \mathrm{C}$. TGA was performed from room temperature (isothermal $5 \mathrm{~min}$ ) to $800^{\circ} \mathrm{C}$ with a heating rate of $10 \mathrm{~K} / \mathrm{min}$ in $\mathrm{N}_{2}$ atmosphere.

\subsubsection{Mechanical tests}

Hardness was measured on a Zwick 8195.04 (according to DIN EN 868, but stacking five $1 \mathrm{~mm}$ thick plates to reach $5 \mathrm{~mm}$ required thickness), repeating the measurements 5 times per sample in random positions. Tensile tests were performed with a Zwick 3150 (Zwick GmbH, Ulm, Germany) with pneumatic clumps (DIN ISO 868) on 6 specimens per measurement whose preparation is described in Sect. 2.2.1.

\section{Results and discussion}

The primary morphological effect obtained by the use of TPUs with different HS/SS composition was on the entity and behaviour of the crystalline and amorphous phase, which were further influenced by the presence of even a small amounts of EG. The different crystallinity of the neat TPUs and the effect of 2 wt\% EG on it was evaluated by DSC (Fig. 1) after melt-processing in the Micro-Compounder. The addition of higher amounts of EG did not significantly change the calorimetry scans, which are not discussed here. First heating was used to unify the thermal history of the samples (DSC data see Table 2).

The cooling run is shown in Fig. 1a. The crystallisation temperature strongly depended on the HS content and has shifted in the presence of EG to a higher temperature, showing the nucleating activity of EG. Observing the crystallization of $\mathrm{hc}-\mathrm{TPU}$, the maximum of the peak $\mathrm{T}_{\mathrm{c}, \mathrm{m}}$ was $152^{\circ} \mathrm{C}$ with an onset temperature of $\mathrm{T}_{\mathrm{c}, \mathrm{o}}$ of $161^{\circ} \mathrm{C}$, which shifted in presence of EG to $T_{c, m}=177^{\circ} \mathrm{C}\left(T_{c, o}=183^{\circ} \mathrm{C}\right)$. The crystallisation occurred in a shorter range of $\mathrm{T}$, which means crystals more homogeneous in dimensions were formed, but the degree of crystallisation was not significantly changed, since the crystallisation enthalpies were similar to hc-TPU and its composites, respectively $-28 \mathrm{~J} / \mathrm{g}$ 
Fig. 1 DSC analysis of neat TPUs (dashed lines) and TPU/ EG (2 wt\%) composites (solid lines): a cooling run; $\boldsymbol{b}$ 2nd heating run
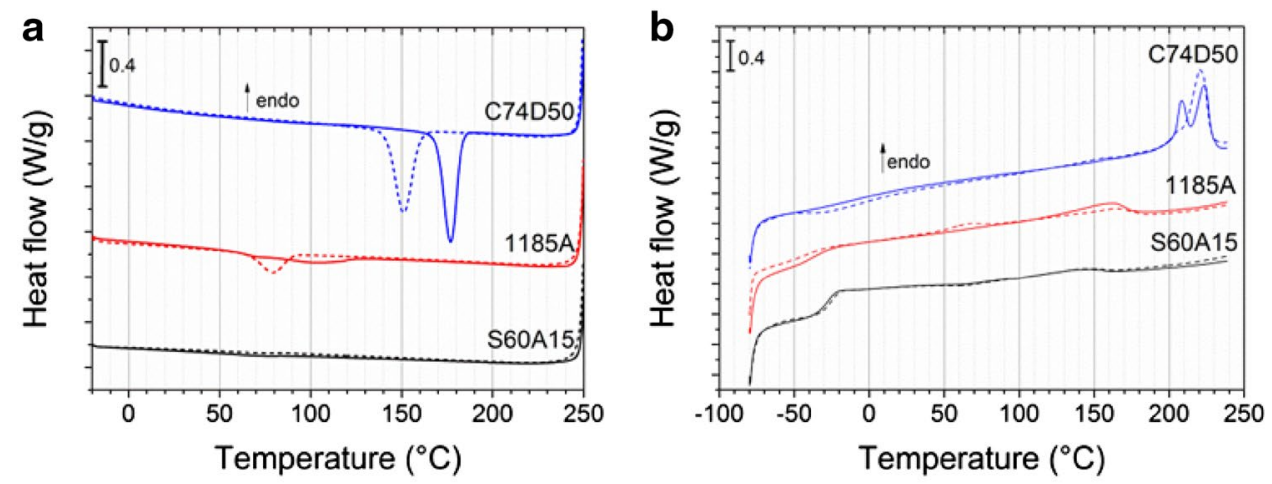

Table 2 Thermal characteristics of the neat TPUs and their composites containing $2 \mathrm{wt} \%$ EG measured with DSC

\begin{tabular}{lllllll}
\hline Material & DSC run & $\mathrm{T}_{\mathrm{g}}\left[{ }^{\circ} \mathrm{C}\right]$ & $\Delta \mathrm{c}_{\mathrm{p}}[\mathrm{J} / \mathrm{g} \mathrm{K}]$ & $\mathrm{T}_{\mathrm{m}}$ or $\mathrm{T}_{\mathrm{c}}\left[{ }^{\circ} \mathrm{C}\right]$ & $\mathrm{T}_{\mathrm{c}, \mathrm{o}}\left[{ }^{\circ} \mathrm{C}\right]$ & $\Delta \mathrm{H}_{\mathrm{m}}$ or $\Delta \mathrm{H}_{\mathrm{c}}[\mathrm{J} / \mathrm{g}]$ \\
\hline Ic-TPU (S60A15) & 1st heat & -34 & 0.28 & $70 / 143$ & - & 8.6 \\
& Cool & n.d. & - & 70 & 82 & -0.5 \\
& 2nd heat & -26 & 0.49 & 140 & - & 3.1 \\
Ic-TPU/EG & 1st heat & -38 & 0.40 & $83 / 149$ & - & 15 \\
& Cool & n.d. & - & 78 & 93 & -1.1 \\
& 2nd heat & -30 & 0.47 & 146 & - & 3.0 \\
mc-TPU (1185A) & 1st heat & n.d. & - & $72 / 163$ & - & 22 \\
& Cool & n.d. & - & 79 & 90 & -9.0 \\
& 2nd heat & -30 & 0.23 & $73 / 145 / 165$ & - & 14 \\
mc-TPU/EG & 1st heat & -40 & 0.24 & $83 / 147$ & - & 27 \\
& Cool & n.d. & - & $70 / 104$ & 132 & -14 \\
& 2nd heat & -34 & 0.34 & 163 & - & 14 \\
hc-TPU (C74D50) & 1st heat & n.d. & - & $55 / 189 / 198 / 210$ & - & 34 \\
& Cool & n.d. & - & 152 & 161 & -28 \\
& 2nd heat & -5 & 0.36 & $157 / 205$ s/221 & - & 26 \\
& 1st heat & n.d. & - & $187 / 197 / 209 / 222$ & - & 26 \\
hc-TPU/EG & Cool & n.d. & - & 177 & 183 & -26 \\
& 2nd heat & -5 & 0.32 & $208 / 223$ & - & 28 \\
\hline
\end{tabular}

and $-26 \mathrm{~J} / \mathrm{g}$ (Table 2). The mc-TPU exhibited a single peak of crystallisation, broader than hc-TPU with $\mathrm{T}_{\mathrm{c}}$ of $79^{\circ} \mathrm{C}$ and $T_{c, O}$ of $90^{\circ} \mathrm{C}$. In the presence of $E G, T_{c, o}$ shifted to $132^{\circ} \mathrm{C}$ and two peaks of crystallization at $104^{\circ} \mathrm{C}$ and $70^{\circ} \mathrm{C}$ appeared, the first attributed to the neat $\mathrm{HS}$ phase, the second to the mixed HS/SS phase. The EG also increased the degree of crystallisation, as shown by the change of $\Delta \mathrm{H}_{c^{\prime}}$ from -9 to $-14 \mathrm{~J} / \mathrm{g}$. Very low crystallisation was observed for Ic-TPU and its composites. The enthalpy was ca. $-0.5 \mathrm{~J} / \mathrm{g}$ for the neat TPU and $-1.1 \mathrm{~J} / \mathrm{g}$ for the composite showing again the nucleation effect of graphite, confirmed by the shift to higher temperatures of $\mathrm{T}_{\mathrm{c},{ }^{\prime}}$, from $82^{\circ} \mathrm{C}$ in the neat polymer to $93^{\circ} \mathrm{C}$ in the composite, while the $\mathrm{T}_{\mathrm{c}}$ remained substantially unaltered and corresponds to the mixed phase HS/ SS.

The increased crystallinity with rising HS content was also apparent in the melting behaviour during the second heating run. Aside from the same chemical structure of the
HS in all TPUs, the melting temperature was highest for the TPU with the highest HS, undoubtedly because of the longer HS length, stronger phase separation and better packing. The hc-TPU exhibited a broad and soft melting at $157^{\circ} \mathrm{C}$ of the crystallite, which formed over the $T_{g}$ and disappeared in the composites. The main melting peak was at $\mathrm{T}_{\mathrm{m}}=221^{\circ} \mathrm{C}$ with a shoulder at a lower temperature around $205^{\circ} \mathrm{C}$. In the composite, the two melting peaks became more defined and did not shift significantly, with maxima at $208^{\circ} \mathrm{C}$ and $223^{\circ} \mathrm{C}$. The multiple melting of TPU was attributed in part to the melting-recrystallization-melting process, and to changes in crystal morphology [31]. The mc-TPU exhibited broad melting regions, of which one at $70^{\circ} \mathrm{C}$ attributed to the mixed phase, which had almost disappeared in the composites. Other broad melting peaks reach maxima at $154^{\circ} \mathrm{C}$ and $165^{\circ} \mathrm{C}$, while the mc-TPU/EG composite had only one melting region with $\mathrm{T}_{\mathrm{m}}=163^{\circ} \mathrm{C}$. The strong reduction of the mixed phase and the presence 
of one single melting peak in the composite, suggested an increase of the phase separation promoted by the filler. In Ic-TPU, both neat and with EG, the higher value of $\Delta \mathrm{H}_{\mathrm{m}}$ than $\Delta \mathrm{H}_{\mathrm{c}}$ suggested an interfering effect by the EG in the melting process, which became negligible in the $\mathrm{mc}$ - and hc-TPU.

In the elastomers used, the $T_{g}$ was not significantly influenced, and moreover the expected effect of an increase due to the lower mobility of the chains was not observed. Indeed, the $\mathrm{T}_{\mathrm{g}}$ of IC-TPU was $-26^{\circ} \mathrm{C}$ and shifted to $-30{ }^{\circ} \mathrm{C}$ in the presence of EG. Similarly, the $\mathrm{T}_{\mathrm{g}}$ of $\mathrm{mc}-\mathrm{TPU}$ moved from $-30{ }^{\circ} \mathrm{C}$ in the neat polymer to $-34{ }^{\circ} \mathrm{C}$ in the composite. However, the $\mathrm{T}_{\mathrm{g}}$ of hc-TPU did not variate and its composite were $-5^{\circ} \mathrm{C}$. In lc- and hc-TPU composites, $\Delta \mathrm{C}_{\mathrm{p}}$ slightly decreased from 0.49 to $0.47 \mathrm{~J} / \mathrm{gK}$ and from 0.36 to $0.32 \mathrm{~J} / \mathrm{gK}$ respectively, compared to the neat polymers, in line with the increase of crystallinity previously observed. On the contrary, in the mc-TPU, $\Delta C_{p}$ increased from 0.23 to $0.34 \mathrm{~J} / \mathrm{gK}$, since the enhanced phase separation resulted on the one hand in better formed crystals in the HS-domain, and on the other hand in an increase of the amorphous phase composed by SS.

The morphology had a determinant influence on the formation of the percolative path and thus on the dependency of resistivity $(\rho)$ on the EG concentration shown in the Fig. 2, where the solid lines illustrate the stages in which the percolative net is completely absent or formed, and the dash line traces the transition between these two states. Therefore it was possible that composites containing the same EG concentration were insulating or conductive. The resistivity $\rho$ of pure polymers differed in the range of 1-2 orders of magnitude and decreased slowly with the increase in EG content when approaching the PC. Between certain EG concentrations, different for any

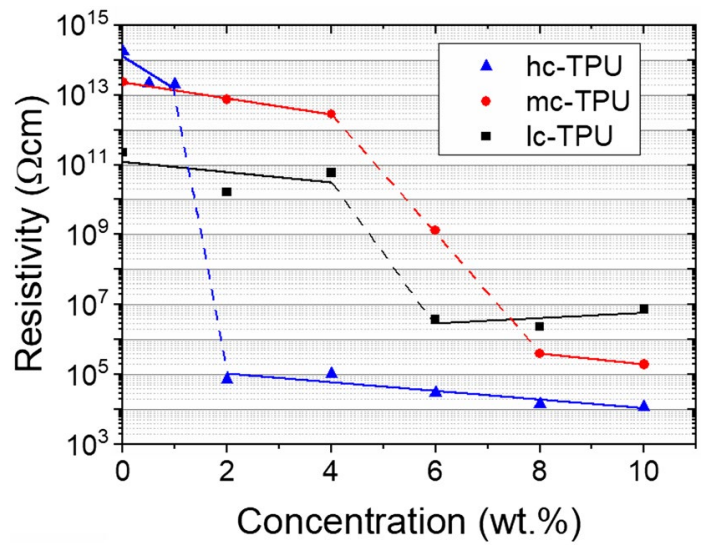

Fig. 2 Electrical resistivity measured at different EG concentrations for the three TPU composite series. Solid lines are linear interpolation of the plateaus before and after the PC, dashed lines are a visual guide
TPU, the values of $\rho$ drops, identifying the concentration ranges in which the $P C$ occurred. It is possible to observe a sharp drop in the case of hc-TPU in-between 1 and 2 wt\% EG and broader ranges in the less crystalline composites. The PC was between 4 and 6 wt $\%$ EG for Ic-TPU composites and about 6 wt $\%$ EG for mc-TPU. Above the percolation a continuous decrease in $\rho$ was observed; the slight increase $\rho$ of Ic-TPU at $10 \mathrm{wt} \%$ can be attributed to the agglomeration of the EG in the soft polymer matrix induced by excess of EG. No strong correlation between PC and SS type and amount could be established (see Table 1) but it was evident that the high crystallinity of hc-TPU played an important role in the formation of conductive paths. At a lower degree of phase separation less crystallinity was detected and higher amounts of EG had to be added to form percolating paths. This indicated that in the phase separated TPU, EG located preferably within the SS domains, and since the volume of this phase was higher in TPU with lower HS contents, more filler was needed to create percolating paths. Good wetting of EG above the percolation by SS may hamper direct contacts between the filler particles and thus electron transport, resulting in lower conductivity of the percolating paths in SS-reach TPUs. If GNP would nucleate the HS crystallisation directly, the crystals at the EG surface would form an isolating layer hindering direct contact between the conductive fillers and so at least hamper the electron transport, but the conductivity of the percolated structures in hc-TPU was higher than in the other TPUs.

The SEM provided a clear explanation for the lower PC of the hc-TPU. On the surface of the cryo-fractured composites a neat parting between the hc-TPU and the EG was observed, emerging naked from the matrix, while in mc-TPU and especially in Ic-TPU the resulting EG flakes were still fully embedded within the polymer (Fig. 3). The SS tendency to wet the filler was attributed to the lower surface tension [32] in competition with the HS, whose higher surface tension was weakly compensated by the tendency to crystallise on the graphite layers. Overall, the EG-flakes were more isolated in the presence of high SS content and more EG was necessary to form an electrical percolative path, as observed for the higher PC of the Ic-TPU and mc-TPU composites. Independently of the used TPU, all composites contained big agglomerates in the $100 \mu \mathrm{m}$ dimension, examples shown for fractured surfaces of compression moulded plates of mc-TPU in Fig. 3d. Thus, the conductive paths were formed by finely dispersed GNP interconnecting agglomerates of different dimensions. A quantification of the degree of agglomeration, which generally depends on the amount of filler and processing conditions [29] was impossible to conduct due to the heterogeneity of the fractured surfaces. 
Fig. 3 SEM analysis of TPU/EG (2 wt\%)-composites, cryofracture surfaces of plates: a Ic-TPU; b mc-TPU; c hc-TPU; $\mathbf{d}$ mc-TPU in lower magnification
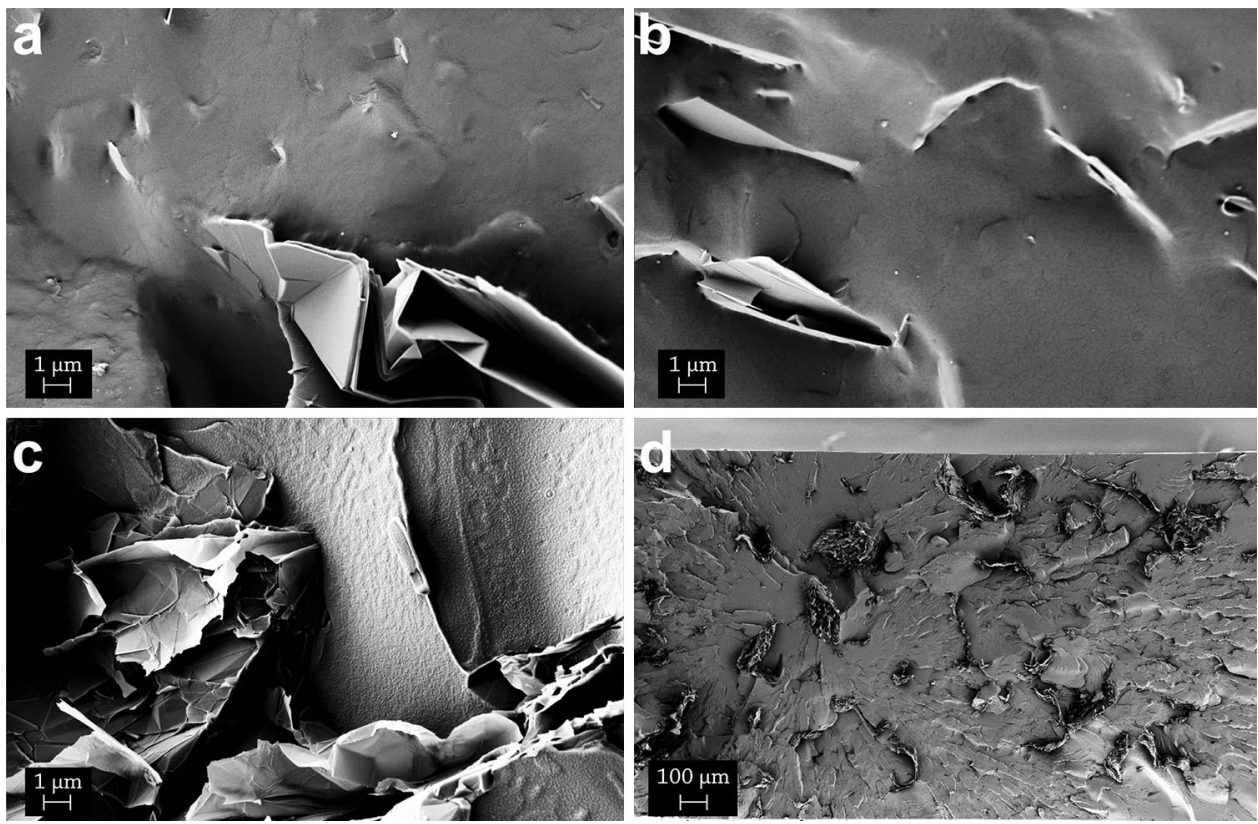

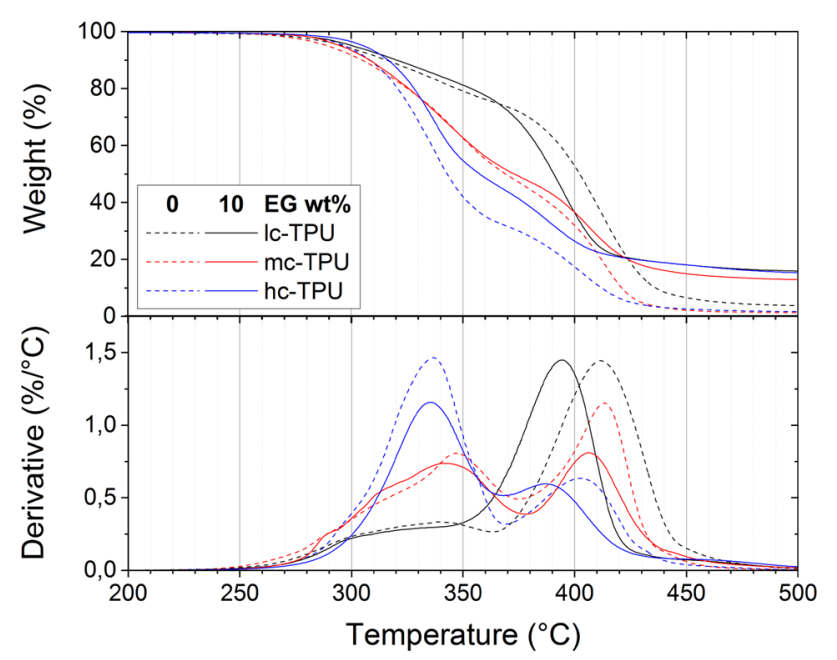

Fig. 4 TGA analysis of pure TPUs (dashed lines) and TPU/EG (10 wt\%) composites (solid lines)

TGA performed on TPUs and TPU/EG-composites reflected not only the HS to SS ratio but also the interaction between the polymer matrix and TPU. In Fig. 4 are plotted the thermogravimetric data of the three TPUs and their composites containing 10 wt\% EG showing their degradation behaviour. At intermediate concentrations the onset, mid-point and completion temperatures are between the two extremes and not influenced by the PC. Composites with lower filler content showed an intermediate behaviour (not shown). In all the samples two main degradation steps were visible. At the temperature range of $300-350^{\circ} \mathrm{C}$, the degradation of HS in two secondary processes occurred, which merged together in the hc-TPU. $\mathrm{HS}$ degraded at lower temperature than SS, since they contained a higher amount of thermally unstable urethane groups [33]. At the higher temperature the SS degraded in a single and well defined process. In the neat polymers, the degradation at high temperature was complete and at every weight loss it was attributed the SS- or HS- domain; it was then possible to estimate the SS/HS weight ratio: ca. 25/75 for hc-TPU, 47/53 for mc-TPU, and 75/25 for lc-TPU. The degradation of HS was not significantly influenced by the presence of EG, while the degradation of SS was shifted to a lower temperature, especially in Ic-TPU containing a softener. This shift could be explained by the fact that EG had higher heat transfer ability than the polymer. The selective effect of EG on the degradation of SS suggested that EG segregated within the SS domains. This was in agreement with the observed percolation phenomena of EG in hc-TPU/EG, where less EG was needed for creating percolation paths due to smaller SS volume fraction compared to TPUs with higher SS fractions. To become conductive, the EG-containing SS domains must form a continuous phase, in which the HS domains are dispersed.

The interaction between the graphitic filler and the polymer matrix was also demonstrated by analyses of the mechanical behaviour, in particular by tensile tests and hardness measurements. The stress-strain curves are shown in Fig. 5a-c. For Ic-TPU and mc-TPUs the deformation behaviour was still elastic for both pure polymers and composites, even if the percolation concentration was reached. The elasticity derives from the elasticity of the SS domains which were physically crosslinked by the HS domains. Introduction of the stiff EG to the TPU reduced 

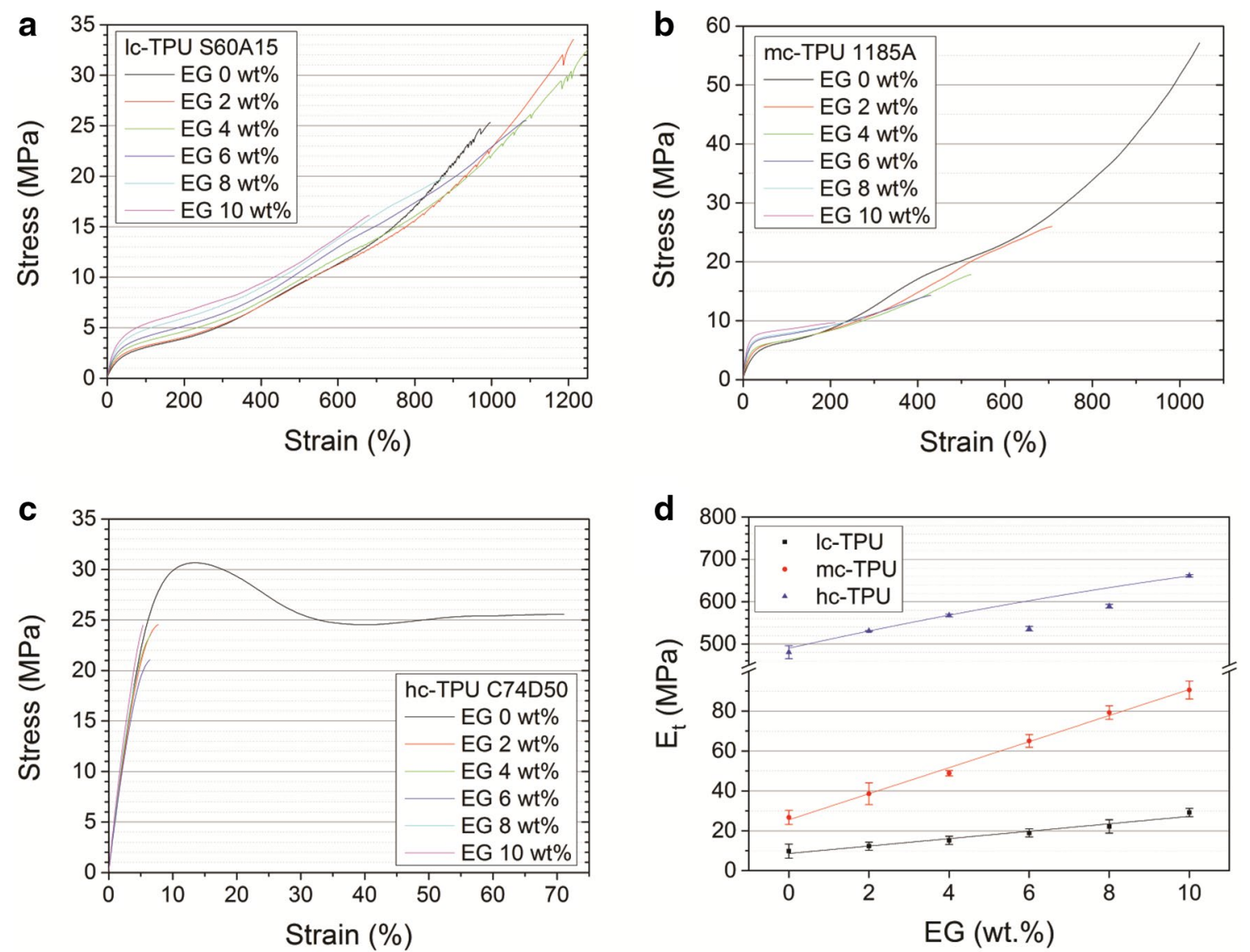

Fig. 5 Deformation behaviour of a Ic-TPU; b mc-TPU; $\mathbf{c}$ hc-TPU and d tensile moduli of the three TPU/EG composite series in dependence on EG concentration. Straight lines are linear extrapolations

the flexibility of the SS, resulting in increased tensile modulus and resistance against deformation. In Ic-TPU composite, at $2 \mathrm{wt} \% \mathrm{EG}$ stress and strain at break increased, and then decreased slightly at $4 \mathrm{wt} \%$, and dropped at $6 \mathrm{wt} \%$, while in mc-TPU composites, they constantly decreased with increasing filler content, because the filler acted as a defect in the matrix. The detrimental filler effect on flexibility became more pronounced as SS fraction in the TPU decreased, because of the relatively higher filler concentration in the SS volume fraction. The pure hc-TPU exhibited an elastic elongation up to $12 \%$ strain followed by a plastic deformation with necking, provoked by shifting of HS crystalline domains until break. The introduction of EG completely suppresses the ductile deformation by blocking the movement of HS domains and thus changing the ductile fracture behaviour to a brittle fracture observed by the drastic drop of stress at break. While the strain at break dropped drastically in the hc-TPU/EG composites, since EG already acted as a catastrophic failure site even at low loadings, the stress at break decreased gradually, because of the reinforcing effect of the filler. The tensile

(values of hc-TPU/EG (6 wt\%) and hc-TPU/EG (8 wt\%) are excluded from interpolation, $\mathrm{R}^{2}=0.999$ )

moduli $E_{t}$ of the three series of composites was calculated by the tangent at the axes origin of the stress-strain curves and summarised in Fig. 5d, exhibiting the strong correlation between tensile strength and HS content. The filler, despite the soft interlayer forces which allow easy deformation of the multilayer structures, opposed deformation, thus increasing the $E_{t}$ of all three TPUs in direct proportion to the EG concentration.

Similar to the elongation tests, the hardness measured on the TPUs and their composites revealed two distinct behaviours. In Fig. 6, the hardness as a dependency of EG concentration was plotted with relative standard deviation and linear interpolation. To satisfy the norm DIN EN 868 was necessary to use two different shores: shore $A$ for the Ic- and mc-TPU (left Y-axe) and shore D for the hc-TPU (right Y-axe). In Ic-TPU and mc-TPUs the hardness increased linearly with the EG concentration, while the hardness of hc-TPU composites was more or less constant in the studied EG concentration range between 0 and $10 \mathrm{wt} \%$. The strongest effect of EG on hardness was observed in the softest matrix (Ic-TPU), while the effect was lower in the 


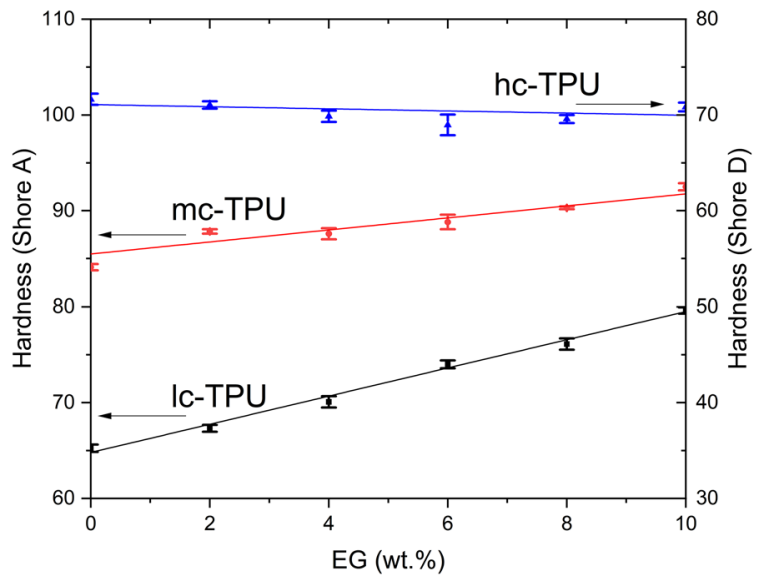

Fig. 6 Hardness in dependency of EG concentration: Ic-TPU and mc-TPU, shore A; hc-TPU, shore D

mc-TPU based composites, which had a higher HS content and crystallinity. In hc-TPU the hardness was much higher than in the other TPU and dominated by the crystalline HS domains. The hardness value of hc-TPU around 70 in shore D corresponds into elastomers to $95-100$ in shore A [34], the addition of EG did not improve it further this value. The small decrease in hardness when adding up to $6 \mathrm{wt} \% \mathrm{EG}$ and soft increase at higher filler loadings may be caused by overlapping effects of the deformability of the EG itself, increased phase separation allowing deformation of the separated SS domain, and changed crystal structure (see DSC, Fig. 1).

Overall, the mechanical analyses showed how combining EG with TPUs of different HS/SS compositions multiply the already broad range of features available from the polyurethanes themselves. By choosing the proper polymer matrix it is possible to obtain tough conductive thermoplastic materials either for antistatic application like coating or floors, or insulating flexible materials with improved flame retardant properties, for example [35].

\section{Conclusions}

Composites based on three TPUs, varying in soft segment structure and HS/SS composition and thus exhibiting different crystallinity, were prepared by melt-mixing with conductive EG. The processing temperatures were chosen so that similar shear forces were obtained when mixing the three different TPUs with EG. Under these conditions, composites prepared form the TPU with the highest HS content and, thus, highest crystallinity (hc-TPU) exhibited the lowest percolation concentration and higher conductivities above the percolation concentration compared to composites prepared from SS rich TPUs (Ic-TPU and mc-TPU). This was due to lower surface tension of SS-domains which wrapped and insulate the graphite nano-flakes. A preferred distribution of the EG inside the SS-domains caused also a stronger phase separation and then a higher amount HS crystallites and lower $\mathrm{T}_{g}$ of the SS; specular to what was observed with graphite functionalised with polar groups, which form hydrogen bonds preferentially with $\mathrm{HS}[36,37]$.

EG improved also the phase separation between the crystalline and the amorphous phase and caused the reduction of the mixed HS/SS domains phase. This phenomenon was also observed with the DSC in particular in the mc-TPU, where the ratio $\mathrm{HS} / \mathrm{SS}$ domains was close to $1 / 1$ estimated by TGA. Thermogravimetric analysis also supported the favourable interaction of EG with SS and its location inside the SS domains, showing the change in the degradation behaviour of SS and changes in mechanical behaviour. The hardness of soft TPUs improved due to the presence of EG while that of hc-TPU, where the HS crystals determine the overall hardness, was nearly unaffected. The ductility of hc-TPU was already changed to brittleness at low EG loadings, since the EG hampers the movement of HS crystallites during elongation and the EG agglomerates act as catastrophic failure sites. In TPUs with higher SS amounts the ductility decreased due to the interaction of SS with EG, but the detrimental effect of EG got lower as the neat TPU was softer. The low interlayer forces between the graphene layers allowed for easy filler deformation and thus relatively good ductility of the Ic-TPU and mc-TPU composites.

Thus, electrically conductive composites with elastomeric character can be produced with the same wt\% of EG and production process, it is then possible to tune ductility, hardness and insulating or conductive behaviour of the composites only by variation of the HS/SS ratio and composition, easily covering a broad range of applications.

Acknowledgements We acknowledge the financial support from Deutsche Forschungsgemeinschaft (DFG; Project PI 308/9-1) and Technology Agency of the Czech Republic (TAČR, project TE01020022). Helfried Kunath is acknowledged for recording SEM and optical images as well as for his general technical assistance. On behalf of all authors, the corresponding author states that there is no conflict of interest.

\section{Compliance with ethical standards}

Conflict of interest The authors declare that they have no competing interests

\section{References}

1. Novoselov KS, Geim AK, Morozov SV, Jiang D, Zhang Y, Dubonos SV, Grigorieva IV, Firsov AA (2004) Electric field effect in atomically thin carbon films. Science 306:666-669 
2. Ebbesen TW, Ajayan PM (1992) Large-scale synthesis of carbon nanotubes. Nature 358:220-222

3. Xie RC, Qu BJ (2001) Synergistic effects of expandable graphite with some halogen-free flame retardants in polyolefin blends. Polym Degrad Stab 71:375-380

4. Galashina NM, Nedorezova PM, Shevchenko VG, Tsvetkova VI, Klyamkina AN, Chmutin IA, Ponomarenko AT, Dyachkovskii FS (1993) Polymerizationally filled electricity and heat-conducting polypropylene graphite composites-some specific features of their properties. Vysokomol Soedin Ser A Ser B 35:A1315-A1319

5. Bledzki AK, Jakobi R (1991) Polyester composite-materials with special properties obtained by matrix modification and additives. Angew Makromol Chem 185:11-21

6. Jang BP, Kowbel W, Jang BZ (1992) Impact behavior and impact-fatigue testing of polymer composites. Compos Sci Technol 44:107-118

7. Saw WPS, Mariatti M (2012) Properties of synthetic diamond and graphene nanoplatelet-filled epoxy thin film composites for electronic applications. J Mater Sci Mater Electron 23:817-824

8. Sharma K, Pareek K, Rohan R, Kumar P (2019) Flexible supercapacitor based on three-dimensional cellulose/graphite/ polyaniline composite. Int J Energy Res 43:604-611

9. Benetti D, Cui DL, Zhao HG, Rosei F, Vomiero A (2018) Direct measurement of electronic band structure in single quantum dots of metal chalcogenide composites. Small 14:1801668

10. Nazir H, Batool M, Osorio FJB, Isaza-Ruiz M, Xu X-H, Vignarooban K, Phelan P, Inamuddin I, Kannan AM (2019) Recent developments in phase change materials for energy storage applications: a review. Int J Heat Mass Transf 129:491-523

11. Pielichowska K, Nowak M, Szatkowski P, Macherzynska B (2016) The influence of chain extender on properties of polyurethane-based phase change materials modified with graphene. Appl Energy 162:1024-1033

12. Kim JT, Kim BK, Kim EY, Park HC, Jeong HM (2014) Synthesis and shape memory performance of polyurethane/graphene nanocomposites. React Funct Polym 74:16-21

13. Park JH, Trung Dung D, Lee H-I, Jeong HM, Kim BK (2014) Properties of graphene/shape memory thermoplastic polyurethane composites actuating by various methods. Materials 7:1520-1538

14. Gilead A (2014) Pure possibilities and some striking scientific discoveries. Found Chem 16:149-163

15. Kim BK, Lee SY, Xu M (1996) Polyurethanes having shape memory effects. Polymer 37:5781-5793

16. Sullivan EM, Oh YJ, Gerhardt RA, Wang B, Kalaitzidou K (2014) Understanding the effect of polymer crystallinity on the electrical conductivity of exfoliated graphite nanoplatelet/polylactic acid composite films. J Polym Res 21:563

17. Rechner T (2004) The uley graphite mine of South Australia. In: Wang Y, Ge S, Guo G (eds) Mining science and technology. CRC Press, Taylor \& Francis Group, Xuzhou, pp 33-38. https://books .google.cz/books?id=OA9Kds-zkUIC\&lpg=PA33\&ots=eCLFS

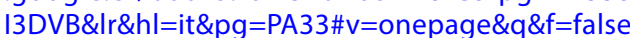

18. Seabra AB, Paula AJ, de Lima R, Alves OL, Duran N (2014) Nanotoxicity of graphene and graphene oxide. Chem Res Toxicol 27:159-168. https://doi.org/10.1021/tx400385x

19. Wang H, Yan H, Zhu M, Liu J, Li S, Zhang S (2010) Preparation of expanded graphite comprises dissolving potassium dichromate in mixed solution of nitric acid and sulfuric acid, adding natural flake graphite, reacting, washing with water, filtering, oven-drying and heating in microwave oven. University of Beijing Technology, CN101891186-A
20. Marcondes Agnelli JA, Marinelli AL, Maciel Miranda A, Finocchio H, Ambrosio JD, Kobayashi M (2012) Method producing polymer expanded graphite nanocomposites used in automobile industry parts like bumpers, mirror boxes and structural components, involves preparing mixtures containing expanded graphite and thermoplastic polymer. University of Fundacao Federal Sao Carlos, BR201004393-A2

21. Gupta A, Goyal RK (2019) Electrical properties of polycarbonate/expanded graphite nanocomposites. J Appl Polym Sci $136: 47274$

22. Redondo-Foj B, Ortiz-Serna P, Carsi M, Jesus Sanchis M, Culebras M, Maria Gomez C, Cantarero A (2015) Electrical conductivity properties of expanded graphite-polycarbonatediol polyurethane composites. Polym Int 64:284-292

23. Lee SH, Kim JH, Kim KW, Youn JR, Choi SH, Kim SY (2009) Effects of filler geometry on internal structure and physical properties of polycarbonate composites prepared with various carbon fillers. Polym Int 58:354-361

24. Zhu D, Bin Y, Matsuo M (2007) Electrical conducting behaviors in polymeric composites with carbonaceous fillers. J Polym Sci Part BPolym Phys 45:1037-1044

25. Nagata K, Iwabuki H, Nigo H (1999) Effect of particle size of graphites on electrical conductivity of graphite/polymer composite. Compos Interfaces 6:483-495

26. Kim H, Miura Y, Macosko CW (2010) Graphene/polyurethane nanocomposites for improved gas barrier and electrical conductivity. Chem Mat 22:3441-3450

27. Pionteck J, Melchor Valdez EM, Piana F, Omastova M, Luyt AS, Voit B (2015) Reduced percolation concentration in polypropylene/expanded graphite composites: effect of viscosity and polypyrrole. J Appl Polym Sci 132:41994

28. Sefadi JS, Luyt AS, Pionteck J, Piana F, Gohs U (2015) Effect of surfactant and electron treatment on the electrical and thermal conductivity as well as thermal and mechanical properties of ethylene vinyl acetate/expanded graphite composites. J Appl Polym Sci 132:42396

29. Piana F, Pionteck J (2013) Effect of the melt processing conditions on the conductive paths formation in thermoplastic polyurethane/expanded graphite (TPU/EG) composites. Compos Sci Technol 80:39-46

30. Taheri S, Sadeghi GMM (2015) Microstructure-property relationships of organo-montmorillonite/polyurethane nanocomposites: influence of hard segment content. Appl Clay Sci 114:430-439

31. Pompe G, Pohlers A, Pötschke P, Pionteck J (1998) Influence of processing conditions on the multiphase structure of segmented polyurethane. Polymer 39:5147-5153

32. Pötschke P, Pionteck J, Stutz H (2002) Surface tension, interfacial tension, and morphology in blends of thermoplastic polyurethanes and polyolefins. Part I. Surface tension of melts of TPU model substances and polyolefins. Polymer 43:6965-6972

33. Gupta T, Adhikari B (2003) Thermal degradation and stability of HTPB-based polyurethane and polyurethaneureas. Thermochim Acta 402:169-181

34. Rogulska M, Podkościelny W, Kultys A, Pikus S, Poździk E (2006) Studies on thermoplastic polyurethanes based on new diphenylethane-derivative diols. I. Synthesis and characterization of nonsegmented polyurethanes from HDI and MDI. Eur Polymer J 42:1786-1797

35. Yao Y-Y, Tian H-F, Yuan L, Wu Q-X, Xiang A-M (2019) Improved mechanical, thermal, and flame-resistant properties of polyurethane-imide foams via expandable graphite modification. J Appl Polym Sci 136:46990 
36. Redondo-Foj B, Ortiz-Serna P, Carsí M, Jesús Sanchis M, Culebras M, Gómez CM, Cantarero A (2014) Electrical conductivity properties of expanded graphite-polycarbonatediol polyurethane composites. Polym Int 64:284-292

37. Razeghi M, Pircheraghi G (2018) TPU/graphene nanocomposites: effect of graphene functionality on the morphology of separated hard domains in thermoplastic polyurethane. Polymer 148:169-180

Publisher's Note Springer Nature remains neutral with regard to jurisdictional claims in published maps and institutional affiliations. 